Pacific Journal of Mathematics

THE DECIDABILITY OF A CLASS OF AE SENTENCE IN THE 


\title{
THE DECIDABILITY OF A CLASS OF $A E$ SENTENCE IN THE ISOLS
}

\author{
Erik Ellentuck and Alfred B. Manaster
}

The isols are a recursive analogue of the Dedekind finite cardinals originally developed by Dekker. In this paper a metatheorem is proved which shows that for certain sentences \& about addition in which no existential quantifier precedes a universal quantifier, the truth of $\mathscr{A}$ in the natural numbers is sufficient to ensure the truth of $\mathscr{A}$ in the isols. A more general class of sentences is also considered and it is seen that the applicability of the metatheorem is also necessary for the truth of any sentence in this class. It follows that there exists a decision procedure for that class of sentences. Extensions of these results to the case of the cosimple isols are also considered.

Careful definitions and exact statements of the results will be given in the next section. The remainder of this section will be devoted to a brief indication of some applications of the metatheorem and to a brief discussion of the relationship of the results in this paper to other work in the theories of addition of the isols, the recursive equivalence types (RETs), and cardinals in set theory without choice. Section 3 contains a proof of the metatheorem. Section 4 contains a proof of the necessity of the metatheorem in the class of sentences referred to above. In $\S 5$ the correctness of the two main results in the cosimple isols is proved.

A typical application of the metatheorem proves the well known refinement property [4, Theorem 14] for the isols.

(1.1) If $A_{1}+\cdots+A_{n}=B_{1}+\cdots+B_{m}$, then there exist isols $C_{i, j}, 1 \leqq i \leqq n, 1 \leqq j \leqq m$ such that

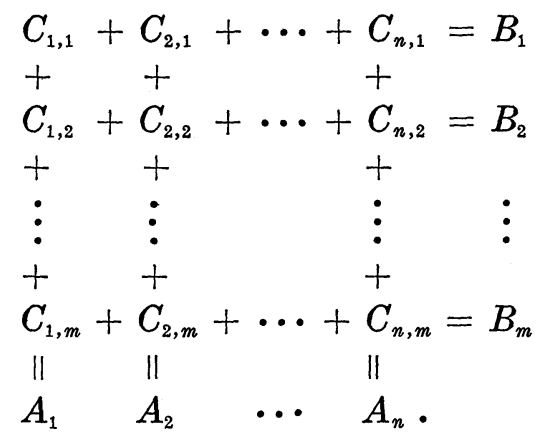

Other examples are the truth in the isols of (1.2) and (1.3).

(1.2) For finite positive $n$ and any $A$ and $B, n A \leqq n B$ implies 
$A \leqq B$

(1.3) For finite positive $n$ and any $A, B$, and $C, n A \leqq B+n C$ implies $A \leqq B+C$.

The truth in the isols of the finite part of Tarski's theorem on the decomposition of linear forms (See [12, Theorem 7] and [13, p. 242].) also follows from the metatheorem. A precise statement of this theorem is given in the next section. The last example is especially interesting because it cannot be extended to the RETs (See Manaster [8].).

The proof of the metatheorem is a generalization of earlier proofs by Dekker-Myhill. It was motivated by these proofs and modifications of them by Friedberg and Nerode. The basic idea of a "chain", which plays a crucial role in the proof of the metatheorem, can be found in Tarski [15] applied to cardinal arithmetic. This type of proof is applied to the isols by Dekker-Myhill in [4] in proving that an isolated set represents an isol and again, in a more complex manner, in proving (1.2) [ibid., Theorems 39 and 40]. Friedberg [6] and Nerode [11] have extended this type of argument to the RETs. It is not possible to extend directly Nerode's results for universal sentences in the RETs to the types of sentences considered here because of the failure of the decomposition of linear forms theorem in the RETs.

An example of an application of the decision procedure is the falsity in the isols of the following sentence, even though it is true in the natural numbers: for every $x_{1}, x_{2}, x_{3}$ there exist $y_{1}$ and $y_{2}$ such that if $5 x_{1}=x_{2}+3 x_{3}$ then $3 x_{1}=5 y_{1}+2 y_{2}$ or $3 x_{2}=5 y_{1}+2 y_{2}$. NerodeManaster [12] shows that while the decision procedure here may be capable of some extension, it can not be extended to a decision procedure for the complete theory of addition of the isols.

The metatheorems proved below were developed at almost the same time as very similar metatheorems were discovered in cardinal arithmetic without the axiom of choice (See Bradford [1].). A significant difference is the restriction to the Dedekind-finite case here. Bradford did not make such a restriction.

2. Preliminaries and summary. The following notational conventions will be used. Some of the notions referred to in this paragraph will be defined later in this section. $E$ denotes the set of natural numbers, $\{0,1,2, \cdots\}$. Lower case English letters, except $f$ and $g$, with or without subscripts, denote natural numbers. $g c d\left(x_{1}, \cdots, x_{n}\right)$ denotes the greatest common divisor of $x_{1}, \cdots x_{n}$. Lower case Greek letters denote sets of natural numbers. $|\xi|$ denotes the cardinality of $\xi$. All applications of pairing functions are implicit so that we identify 
an $n$-tuple of natural numbers with a natural number. $\xi^{j, i}$ denotes $\xi \times\{j\} \times\{i\}=\{(x, j, i) \mid x \in \xi\}$. The letters $X, Y, Z, V$, with or without subscripts or superscripts, denote formal variables and, at times, isols or natural numbers. The letter $A$, with or without subscripts, denotes an indecomposable isol. Vector notation may be used; thus $X$ denotes $\left(X_{1}, \cdots, X_{n}\right)$ for some $n$. Any conjunction, disjunction, or summation is finite even if the range of the operator is not explicitly indicated. The union over an empty index set is empty; thus $\bigcup_{0<k \leqq 0} \delta_{k}=\varnothing$. The matrix of a formula is the formula remaining after the initial string of quantifiers is removed.

Two sets $\alpha$ and $\beta$ are called recursively equivalent if there exists a 1-1 partial recursive function $f$ such that the domain of $f$ includes $\alpha$ and the image of $\alpha$ under $f$ is $\beta$. Recursive equivalence is an equivalence relation on the power set of $E$. The equivalence classes under this relation are called recursive equivalence types (RETs). The RET to which the set $\alpha$ belongs is denoted $\langle\alpha\rangle$.

A set $\xi$ is isolated if it does not contain any infinite recursively enumerable (r.e.) subset. An RET $X$ is called an isol if any $\xi \in X$ is isolated. $\Delta$ denotes the set of isols. $\xi$ and $\eta$ are called recursively separated if there exist disjoint r.e. sets $\omega$ and $\theta$ such that $\xi \subseteq \omega$ and $\eta \subseteq \theta$. The sum of $X$ and $Y$ is defined to be $\langle\xi \cup \eta\rangle$ where $\xi \in X, \eta \in Y$, and $\xi$ and $\eta$ are recursively separated. If $\xi$ and $\eta$ are recursively separated we may write $\xi \cup \eta$ as $\xi+\eta . X$ is said to be less than or equal to $Y(X \leqq Y)$ if there is a $Z$ such that $X+Z=Y$. Basic properties of the RETs and the isols may be found in [4]. In particular, the isols satisfy the additive cancellation law,

$$
(\forall X)(\forall Y)(\forall Z)[X+Y=X+Z \longrightarrow Y=Z] \text { • }
$$

An infinite isol, $A$, is called indecomposable if $A=X+Y$ implies $X$ is finite or $Y$ is finite. The existence of uncountably many pairwise incomparable indecomposable isols is well known (See Dekker [3, T1] or Dekker-Myhill [4, p. 103].). Define $X={ }_{1} Y$ to mean there exist finite $m$ and $n$ such that $X+m=Y+n$. Generalizations of indecomposability and $=_{1}$ are considered in Manaster [9]. The following properties will be used in $\S 4$.

(2.2) If $A$ is indecomposable and $A=\sum X_{i}$, then there is exactly one $i$ for which $X_{i}$ is infinite; moreover for that $i, X_{i}={ }_{1} A$ and so $X_{i}$ is indecomposable.

(2.3) If $X={ }_{1} Y$, then $X$ and $Y$ are comparable.

(2.4) If $A, A_{1}, \cdots, A_{n}$, are indecomposable and $A \leqq \sum A_{i}+p$, then for some $i, A={ }_{1} A_{i}$.

(2.5) If $A_{1}, \cdots, A_{m}$ are pairwise incomparable indecomposable isols, 
then there is no isol $V$ and $j, 1 \leqq j \leqq m$, such that $2 V \leqq \sum A_{k} \& A_{j} \leqq$ $3 V$.

Proofs. (2.2) is proved by a routine induction on the number of summands. To prove (2.3) suppose $X={ }_{1} Y$. Let $X+m=Y+n$. If $m \geqq n$, then (2.1) shows $X+(m-n)=Y$ and hence $X \leqq Y$. A symmetric argument shows that if $m<n, Y \leqq X$.

For (2.4) suppose $A, A_{1}, \cdots, A_{n}$ are indecomposable and $A \leqq$ $\sum A_{i}+p$. The refinement property gives the existence of $X_{1}, Y_{1}, \cdots$, $X_{n+1}, Y_{n+1}$ such $X_{i}+Y_{i}=A_{i}$ for $i \leqq n, X_{n+1}+Y_{n+1}=p, A=\sum X_{i}$, and $Y=\sum Y_{i}$. (2.2) gives the existence of a unique $i$ such that $A={ }_{1} X_{i}$. Since $X_{i}$ is infinite, $i \leqq n$. A further application of $(2.2)$ to $X_{i}+Y_{i}=A_{i}$ gives $X_{i}={ }_{1} A_{i}$. Thus $A={ }_{1} A_{i}$.

To prove (2.5) suppose $A_{1}, \cdots, A_{m}$ are pairwise incomparable indecomposable isols, $2 V \leqq \sum A_{k}$, and $A_{j} \leqq 3 V$ for some $j, 1 \leqq j \leqq m$. Since $A_{j} \leqq 3 V=V+V+V$, the refinement property and (2.2) combine to show that there is a natural number $q$ such that $A_{j} \leqq V+q$. Thus $2 A_{j} \leqq 2 V+2 q \leqq \sum A_{k}+2 q$. (2.1) shows $A_{j} \leqq \sum_{k \neq j} A_{k}+2 q$. (2.4) then shows $A_{j}={ }_{1} A_{k}$ for some $k \neq j$. Thus, by (2.3), $A_{j}$ is comparable with $A_{k}$.

An $A E$ sentence is a sentence with a prenex form in which no existential quantifier precedes any universal one. " $E \models \mathscr{A}$ " asserts that $\mathscr{A}$ is true in the natural numbers. " $\Lambda \vDash \mathscr{A}$ " asserts that $\mathscr{A}$ is true in the isols. The following result is the main result of this paper:

$A E$ metatheorem. Let $\mathscr{F}$ be any sentence of the following form:

$$
\begin{gathered}
\left(\forall X_{1}\right) \cdots\left(\forall X_{p}\right)\left(\exists Y_{1}\right) \cdots\left(\exists Y_{r}\right)\left[\&_{j=1}^{n}\left(\sum_{i=1}^{p} a_{j, i}^{\prime} X_{i}=\sum_{i=1}^{p} a_{j, i}^{\prime \prime} X_{i}\right)\right. \\
\left.\longrightarrow \&_{j=1}^{m}\left(\sum_{i=1}^{p} b_{j, i}^{\prime} X_{i}+\sum_{i=1}^{r} c_{j, i}^{\prime} Y_{i}=\sum_{i=1}^{p} b_{j^{\prime}, i}^{\prime \prime} X_{i}+\sum_{i=1}^{r} c_{j, i}^{\prime \prime} Y_{i}\right)\right] .
\end{gathered}
$$

If $E \vDash \mathscr{F}$, then $\Lambda \vDash \mathscr{F}$.

An isol analogue of a theorem originally proved by Tarski for the theory of cardinal numbers without the axiom of choice follows. Its proof (See [8].) led to the discovery of the $A E$ metatheorem.

Theorem on the decomposition of linear forms. Let $X_{1}, \cdots, X_{m}$ be isols satisfying the system of linear equations:

$$
\sum_{k=1}^{n}\left(\sum_{i=1}^{m} \alpha_{k, i}^{\prime} X_{i}=\sum_{i=1}^{m} \alpha_{k, i}^{\prime \prime} X_{i}\right) \text {. }
$$

There exist isols $Y_{1,1}, Y_{1,2}, Y_{2,1}, Y_{2,2}, Y_{3,1}, Y_{3,2}, \cdots, Y_{m, 1}, Y_{m, 2}$ such that $X_{i}=Y_{i, 1}+Y_{i, 2}$ for $i=1, \cdots, m, Y_{1,1} \leqq Y_{2,1}, Y_{2,2} \leqq Y_{1,2}$, and both $Y_{1,1}$, $Y_{2,1}, \cdots, Y_{m, 1}$ and $Y_{1,2} Y_{2,2}, \cdots, Y_{m, 2}$ satisfy the original system of equations (i.e., both $\&\left(\sum a_{k, i}^{\prime} Y_{i, 1}=\sum \alpha_{k, i}^{\prime \prime} Y_{i, 1}\right)$ and $\left.\&\left(\sum a_{k, i}^{\prime} Y_{i, 2}=\sum \alpha_{k, i}^{\prime \prime} Y_{i, 2}\right)\right)$. 
The conclusion of the theorem on the decomposition of linear forms can be strengthened.

Extension of linear forms decomposition theorem. Let $X_{1}, \cdots, X_{m}$ satisfy (2.7). Let $g_{1}, \cdots, g_{m !}$ be the set of permutations on $\{1, \cdots, m\}$. Then there exist isols $X_{i, j}$ for $1 \leqq i \leqq m, 1 \leqq j \leqq m !$ such that

$$
\begin{gathered}
X_{i}=\sum_{j=1}^{m !} X_{i, j} \quad \text { for } \quad i=1, \cdots, m, \\
\&\left(\sum a_{k, i}^{\prime} X_{i, j}=\sum a_{k, j}^{\prime \prime} X_{i, j}\right) \quad \text { for } \quad j=1, \cdots, m !,
\end{gathered}
$$

and $g_{j}(i)<g_{j}(h)$ implies $X_{i, j} \leqq X_{h, j}$ for $i, h \in\{1, \cdots, m\}$ and $1 \leqq j \leqq m$.

The other metatheorem, providing a partial converse to the $A E$ metatheorem, is most easily stated after two definitions are given.

Definition. A special $A E$ sentence is any sentence in the form:

$$
\begin{aligned}
& \left(\forall X_{1}\right) \cdots\left(\forall X_{p}\right)\left(\exists Y_{1}\right) \cdots\left(\exists Y_{r}\right)\left[\&_{k=1}^{n}\left(\sum_{i=1}^{p} a_{k, i}^{\prime} X_{i}=\sum_{i=1}^{p} a_{k, i}^{\prime \prime} X_{i}\right)\right. \\
& \left.\longrightarrow \bigvee_{j=1}^{m}\left(\sum_{h=1}^{q_{j}}\left(\sum_{i=1}^{p} b_{j, h, i}^{\prime} X_{i}+\sum_{i=1}^{r} c_{j, h, i}^{\prime} Y_{i}=\sum_{i=1}^{p} b_{j, h, i}^{\prime \prime} X_{i}+\sum_{i=1}^{r} c_{j, h, i}^{\prime \prime} Y_{i}\right)\right)\right] .
\end{aligned}
$$

A Horn-reduct of the sentence (2.8) is one of the $m$ sentences obtained by deleting all but one of the disjuncts in the consequent of the matrix of the (2.8); thus it is one of the $m$ formulas

$$
\begin{aligned}
& \left(\forall X_{1}\right) \cdots\left(\forall X_{p}\right)\left(\exists Y_{1}\right) \cdots\left(\exists Y_{r}\right)\left[\&\left(\sum a_{k, i}^{\prime} X_{i}=\sum a_{k, i}^{\prime \prime} X_{i}\right)\right. \\
& \left.\quad \longrightarrow{ }_{h=1}^{q_{j}}\left(\sum b_{j, h, i}^{\prime} X_{i}+\sum c_{j, h, i}^{\prime} Y_{i}=\sum b_{j, h, i}^{\prime \prime} X_{i}+\sum c_{j, h, i}^{\prime \prime} Y_{i}\right)\right] .
\end{aligned}
$$

A converse to the $A E$ metatheorem. A special $A E$ sentence is true in $A$ only if one of its Horn-reducts is true in $E$.

Since the $A E$ metatheorem implies the converse, a special $A E$ sentence is true in $A$ iff one of its Horn-reduct is true in $E$. Presburger [13] gives an effective procedure for determining the truth or falsity of each of the Horn-reducts of (2.8) in E. Thus Presburger's result together with the equivalence just stated gives a decision procedure for special $A E$ sentences in the theory of addition of isols.

3. The $A E$ metatheorem. Let $\mathscr{F}$ be the sentence (2.6). The antecedent, or hypothesis, of the matrix of $\mathscr{F}$ will be denoted by $H$, the consequent by $C$. Since both $E$ and $A$ satisfy the additive concellation law, we may assume with no loss of generality that

$$
\min \left(a_{j, i}^{\prime}, a_{j, i}^{\prime \prime}\right)=\min \left(b_{j, i}^{\prime}, b_{i, i}^{\prime \prime}\right)=\min \left(c_{j, i}^{\prime}, c_{j, i}^{\prime \prime}\right)=0 .
$$

(Explicit mention of the range of subscripts will be omitted; the range 
is always finite and determined by $\mathscr{F}$ in an appropriate way.)

Assume $E \vDash \mathscr{F}$. Assume further that $\bar{X}$ is a vector of isols satisfying $H$. For each $i$ let $\xi_{i}$ be a representative of $X_{i}$ where there exist pairwise disjoint r.e. sets $\omega_{i}$ such that each $\xi_{i} \subseteq \omega_{i}$ and $\bigcup \omega_{i} \subseteq$ $\{2 x \mid x \in E\}$. Since $\bar{X}$ satisfies $H$, there exists a set of 1-1 partial recursive functions $f_{j}$ such that for each $j$ the domain of $f_{j}$ includes $\sum_{i}\left(\sum_{0<k \leqq a_{j, i}^{\prime}} \xi_{i}^{j, k}\right)$ and

$$
f_{j}\left(\sum_{i} \sum_{0<k \leqq a_{j, i}} \xi_{i}^{j, k}\right)=\sum_{i} \sum_{0<k \leqq a_{j^{\prime}, i}^{\prime}} \xi_{i}^{j, k}
$$

To prove the theorem a method of enumerating 1-1 partial recursive functions $g_{j}$ must be described and sets $\eta_{i}$ must be defined such that for each $j$ the domain of $g_{j}$ includes $\sum_{i}\left(\sum_{0<k \leqq b_{j, i}^{\prime} \xi_{i}^{j, k}}\right)+\sum_{i} \sum_{0<k \leqq c_{j, i}^{\prime}} \eta_{i}^{j, k}$ and

$$
g_{j}\left(\sum_{i}\left(\sum_{0<k_{k} \leq b_{j, i}^{\prime}, i} \xi_{i}^{j, k}\right)+\sum_{i} \sum_{0<k \leq c_{j, i}^{\prime}} \eta_{i}^{j, k}\right)=\sum_{i}\left(\sum_{0<k \leq b_{j, i}^{\prime \prime}} \eta_{i}^{j, k}\right)+\sum_{i} \sum_{0<k \leq c_{j, i}^{\prime}} \eta_{i}^{j, k} .
$$

Let

$$
a_{j, i}=\max \left(a_{j, i}^{\prime}, a_{j, i}^{\prime \prime}\right), b_{j, i}=\max \left(b_{j, i}^{\prime}, b_{j, i}^{\prime \prime}\right), c_{j, i}=\max \left(c_{j, i}^{\prime}, c_{j, i}^{\prime \prime}\right) .
$$

If $x \in \omega_{i},(x, j, k)$ is called an $H$-version of $x$ if $0<k \leqq a_{j, i}$ and $(x, j, k)$ is called a $C$-version of $x$ if $0<k \leqq b_{j, i}$. If $x \in \omega_{i}, y \in \omega_{i^{\prime}},(x, j, k)$ is an $H$-version of $x$ and $\left(y, j, k^{\prime}\right)$ an $H$-version of $y,(x, j, k)$ is said to be $H$-satisfied by $\left(y, j, k^{\prime}\right)$ and conversely in case $a_{j, i}^{\prime}>0, a_{j, i^{\prime}}^{\prime \prime}>0$, and $f_{j}(x, j, k)=\left(y, j, k^{\prime}\right) . \quad x$ and $y$ are directly $H$-linked if there are $H$ versions of $x$ and $y$ which satisfy each other. $x$ is said to be $H$ linked to $y$ if $x \in \sum \omega_{i}$ and $x=y$ or if there is a finite sequence $x=x_{1}, \cdots, x_{n}=y$ such that each $x_{i}$ is directly $H$-linked to $x_{i+1}$. An $H$-chain is a subset of $\sum \omega_{i}$ any two elements of which are $H$-linked to each other. A complete $H$-chain is an $H$-chain in which all $H$-versions of the elements of the chain are $H$-satisfied by $H$-versions of elements of the chain.

Observe that the relation of being $H$-linked is an equivalence relation on $\sum \omega_{i}$ so that the set of maximal $H$-chains partitions $\sum \omega_{i}$. Since each $f_{j}$ is partial recursive and each $\omega_{i}$ is r.e., each maximal $H$-chain is r.e. To enumerate the set of elements linked to a given $x, x \in \sum \omega_{i}$, simultaneously enumerate the sets $\omega_{i}$ and the functions $f_{j}$. Whenever sufficient information has been generated to show that $y$ is $H$-linked to $x$, place $y$ in the $H$-chain defined by $x$. It can be proved by induction on the number of direct $H$-links required to link $y$ to $x$ that any $y$ which is $H$-linked to $x$ will be placed in the enumerated $H$-chain. If $x$ belongs to a finite complete $H$-chain, there is 
a finite stage of the enumeration at which this complete $H$-chain is enumerated. At some, possibly later, finite stage of the process all information needed to show that the $H$-chain is complete will have been generated since all $H$-versions of all elements of the chain will have been satisfied by $H$-versions of other elements of the chain.

Suppose that $\gamma$ is a finite complete $H$-chain. Let $\gamma_{i}=\gamma \cap \omega_{i}$ and let $x_{i}=\left|\gamma_{i}\right|$. The functions $f_{j}$ show that $\bar{x}$ satisfies $H$. Since $\mathscr{F}$ is true in $E$, there exist natural numbers $\bar{y}$ such that $(\bar{x}, \bar{y})$ satisfies $C$. Moreover a sequence of numbers $\bar{y}$ with this property can be found effectively. Let $\bar{\delta}$ be a sequence of pairwise disjoint sets of odd

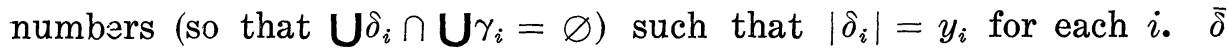
may also be chosen in an effective way. For the remainder of this paragraph let the notions of $C$-versions of elements of $\mathbf{U} \delta_{i}$ be defined analogously to that for elements of $\bigcup \omega_{i}$ : in detail, $(x, j, k)$ is a $C$ version of $x \in \delta_{i}$ if $0<k \leqq c_{j, i}$. There is an effective procedure for defining 1-1 partial functions $g_{j}$ such that, with the obvious definitions relative to the functions $g_{j}, \bigcup \gamma \cup \cup \delta_{i}$ is a finite complete $C$-chain. This effective procedure exists because, for each $j$,

$$
\begin{aligned}
& =\left|\sum_{i}\left(\sum_{0<k \leqq b_{j, i}^{\prime}} \gamma_{i}^{j, k}\right)+\sum_{i} \sum_{0<k \leq c_{j, i}^{\prime}} \delta_{i}^{j, k}\right| \\
& =\sum_{i} b_{j, i}^{\prime} x_{i}+\sum c_{j, i}^{\prime} y_{i}=\sum_{i} b_{j, i}^{\prime \prime} x_{i}+\sum_{i} c_{j, i}^{\prime \prime} y_{i} \\
& =\left|\sum_{i}\left(\sum_{0<k \leqq b_{j, i}^{\prime \prime}} \gamma_{i}^{j, k}\right)+\sum_{i} \sum_{0<k \leq c_{j, i}^{\prime}} \delta_{i}^{j, k}\right| .
\end{aligned}
$$

The constructions of the preceding paragraphs may be combined to enumerate sets $\zeta_{i}$ and functions $g_{j}$ in the following way. By simultaneously enumerating all maximal $H$-chains and checking to see when a finite complete $H$-chain has been enumerated, an effective enumeration of all finite complete $H$-chains is given. Whenever a finite complete $H$-chain is enumerated and identified, find sets $\bar{\delta}$ and functions $g_{j}$ as in the preceding paragraph and, further, such that $\mathbf{U} \delta_{i}$ is disjoint from the union of all the sets chosen as $\delta_{i}$ 's at earlier stages of the enumeration. For each $i$, let $\zeta_{i}$ be the union of all the sets $\delta_{i}$ enumerated in the process. For each $j$ let $g_{j}$ be the union of all the finite functions enumerated as $g_{j}$ 's. Each $g_{j}$ is a 1-1 partial recursive function and $\bar{\zeta}$ is a sequence of pairwise disjoint r.e. sets. Now define $C$-chain and related notions analogously to the corresponding definitions for $H$; here elements of $\sum \omega_{i}+\sum \zeta_{i}$ will be considered and the functions $g_{j}$ will be used. The construction has the property that any finite complete $H$-chain is contained in a finite complete $C$-chain formed by adjoining elements of $\bigcup \delta_{i}$ for sets $\delta_{i}$ such that for each $i, \delta_{i} \leqq \zeta_{i}$.

For each $i$ define $\eta_{i}$ to be the subset of $\zeta_{i}$ consisting of elements 
$C$-linked to some element of $\sum \xi_{i}$. Observe that if $x \in \sum \xi_{i}$ and $y$ is $H$-linked to $x$, then $y \in \sum \xi_{i}$. This may be proved by an induction on the number of links in an $H$-chain from $x$ to $y$ using (3.1). Therefore, since the even elements of any complete $C$-chain are precisely the elements of some finite complete $H$-chain, the even elements of any complete $C$-chain are either all in $\sum \xi_{i}$ or none in $\sum \xi_{i}$. Finally a finite complete $C$-chain is either a subset of $\sum \xi_{i}+\sum \eta_{i}$ or disjoint from this set.

Each $\eta_{i}$ is isolated. Since any element of $\eta_{i}$ is $C$-linked to at least one element of $\sum \xi_{i}$, this follows from the existence of a simultaneous effective enumeration of all complete $C$-chains (in fact, the procedure defining $\bar{g}$ and $\bar{\zeta}$ is, essentially, such an enumeration). Thus, if $\eta$ were an infinite r.e. subset of $\eta_{j}$, we could obtain from its enumeration and the enumeration of all complete $C$-chains an infinite r.e. subset of $\sum \xi_{i}$ contradicting the assumption that each $\xi_{i}$ is isolated.

The critical observation now is that if $x \in \sum \xi_{i}$, then $x$ belongs to a finite complete $H$-chain. An induction shows that if $x \in \sum \xi_{i}$, then $x$ does belong to a complete $H$-chain. If this complete $H$-chain were infinite, it would be an infinite r.e. subset of $\sum \xi_{i}$, again contradicting the assumption that each $\xi_{i}$ is isolated. Since any finite complete $H$-chain is contained in a finite complete $C$-chain, any $x \in$ $\sum \xi_{i}$ belongs to a finite complete $C$-chain which is a subset of $\sum \xi_{i}+\sum \eta_{i}$. This completes the proof.

The two theorems on the decomposition of linear forms are immediate consequences of the $A E$ metatheorem. For each system of equations (2.7), each theorem can be expressed as a sentence true in $E$ to which the metatheorem applies.

4. A converse to the $A E$ metatheorem. The proof of the converse to the $A E$ metatheorem described in $\S 2$ begins with an observation about the natural numbers which was exploited by Bradford in [1].

LEMMA.

$$
\begin{aligned}
E & \vDash(\forall X)\left(\forall Y_{1}\right) \cdots\left(\forall Y_{n}\right)\left[7\left(X=1 \& \&_{i=1}^{n} Y_{i}=0\right)\right. \\
& \left.\longleftrightarrow(\exists V)\left[2 V \leqq X+\sum_{i=1}^{n} Y_{i} \& X \leqq 3 V\right]\right] .
\end{aligned}
$$

Proof. Let $X, Y_{1}, \cdots, Y_{n}$ be any natural numbers. Suppose first there is a $V$ satisfying the matrix of the second part of the equivalence. If $V=0$, then $X=0$ and the first part of the equivalence is also satisfied. If $V>0$, then $X+\sum Y_{i} \geqq 2$. Thus $X \geqq 2$ or $\sum Y_{i}>$ 
0 so $X \geqq 2$ or at least one $Y_{i}>0$. In any event, the first part of the equivalence is satisfied.

For the converse consider first $X$ even. In this case $V=(1 / 2) X$ will satisfy the matrix of the second part of the equivalence. If $X$ is odd but different from 1 , then $V=1 / 2(X-1)$ satisfies the matrix since $2 V=X-1 \leqq X \leqq 3 / 2(X-1)$. Finally if $X$ is 1 but some $Y_{i}>$ $0, V=1$ satisfies the matrix.

Let $\mathscr{F}$ be the formula (2.8) and let $\mathscr{F}_{j}$ be the formula (2.9) for $1 \leqq j \leqq m$. Let $\mathscr{H}\left(X_{1}, \cdots, X_{p}\right)$ be the hypothesis of the matrix of (2.8) and let $\mathscr{C}_{j}\left(X_{1}, \cdots, X_{p}, Y_{1}, \cdots, Y_{r}\right)$ be the conclusion of the matrix of $\mathscr{F}_{j}$. To prove the converse, assume $E \neq \mathscr{F}_{j}$ for $j=1, \cdots, m$. For each $j=1, \cdots, m$, let $\bar{x}_{j}=\left(x_{j, 1}, \cdots, x_{j, p}\right)$ be a sequence of natural numbers such that

$$
E \vDash \mathscr{C}\left(x_{j, 1}, \cdots x_{j, p}\right)
$$

but

$$
E \vDash>\left(\exists Y_{1}\right) \cdots\left(\exists Y_{r}\right) \mathscr{C}_{j}\left(x_{j, 1}, \cdots, x_{j, p}, Y_{1}, \cdots, Y_{r}\right) .
$$

Since $\mathscr{H}$ is a conjunction of homogeneous linear equations and any linear combination of solutions to a system of homogeneous equations is itself a solution to the system,

$$
E \vDash\left(\forall Z_{1}\right) \cdots\left(\forall Z_{m}\right) \mathscr{H}\left(\sum_{j=1}^{m} x_{j, 1} Z_{j}, \cdots, \sum_{j=1}^{m} x_{j, p} Z_{j}\right) .
$$

An immediate consequence of (4.2) is

$$
\begin{gathered}
E \vDash\left(\forall Z_{1}\right) \cdots\left(\forall Z_{m}\right)\left(\forall Y_{1}\right) \cdots \\
\left(\forall Y_{r}\right)\left[\mathscr{C}_{j}\left(\sum_{k=1}^{m} x_{k, 1} Z_{k}, \cdots, \sum_{k=1}^{m} x_{k, p} Z_{k}, Y_{1}, \cdots, Y_{r}\right)\right. \\
\left.\longrightarrow>\left(Z_{j}=1 \& \underset{k \neq j}{\&} Z_{k}=0\right)\right] .
\end{gathered}
$$

Applying the equivalence of Lemma 4.1 to the conclusion of the matrix, using the definition of $\leqq$, and extracting quantifies gives

$$
E \vDash\left(\forall Z_{1}\right) \cdots\left(\forall Z_{m}\right)\left(\forall Y_{1}\right) \cdots\left(\forall Y_{r}\right)(\exists V)\left(\exists V_{0}\right)\left(\exists V_{1}\right)[\mathscr{C l}]
$$

where $\mathscr{M}$ is

$$
\begin{aligned}
& \mathscr{C}_{j}\left(\sum x_{k, 1} Z_{k}, \cdots, \sum x_{k, p} Z_{k}, Y_{1}, \cdots, Y_{r}\right) \\
& \longrightarrow\left(2 V+V_{0}=\sum Z_{k} \& Z_{j}+V_{1}=3 V\right) .
\end{aligned}
$$

Now apply the $A E$ metatheorem to see that the formulas in (4.3) and (4.5) are true in the isols.

Let $A_{1}, \cdots, A_{m}$ be pairwise incomparable indecomposable isols. Let 
$X_{1}=\sum_{k=1}^{m} x_{k, 1} A_{k}, \cdots, X_{p}=\sum_{k=1}^{m} x_{k, p} A_{p}$. By (4.3) in $\Lambda, \Lambda \vDash \mathscr{H}\left(X_{1}, \cdots, X_{p}\right)$. Assume now that $A \vDash \mathscr{F}$. Since $\bar{X}$ satisfies the hypothesis of $\mathscr{F}$ there must be a $j, 1 \leqq j \leqq m$, and isols $Y_{1}, \cdots, Y_{r}$ such that $\Lambda \vDash$ $\mathscr{C}_{j}\left(X_{1}, \cdots, X_{p}, Y_{1}, \cdots, Y_{r}\right)$. Recalling the definition of $X$,

$$
\Lambda \vDash \mathscr{C}_{j}\left(\sum x_{k, 1} A_{k}, \cdots, \sum x_{k, p} A_{k}, Y_{1}, \cdots, Y_{r}\right) .
$$

By (4.5) in $A, A \models(\exists V)\left[2 V \leqq \sum A_{k} \& A_{j} \leqq 3 V\right]$. Since $A_{1}, \cdots, A_{m}$ are pairwise incomparable, this contradicts (2.5). Thus if $E \notin \mathscr{F}_{j}$ for $j=1, \cdots, m$, then $\Lambda \mid \neq \mathscr{F}$.

It is perhaps of interest to point out an alternative to the last paragraph. Using the truth in $\Lambda$ of (4.3), (4.5), and $\mathscr{F}$, it is possible to show $A \vDash\left(\forall Z_{1}\right) \cdots\left(\forall Z_{m}\right)(\exists V)\left[\mathrm{V}_{j=1}^{m}\left(2 V \leqq \sum Z_{k} \& Z_{j} \leqq 3 V\right)\right]$. However this is not the case since if $Z_{1}, \cdots, Z_{m}$ is a sequence of pairwise incomparable indecomposable isols, there is no $V$ satisfying the matrix.

5. The $\boldsymbol{A E}$ metatheorem for cosimple isols. An isol $X$ is cosimple if it has a representative $\xi$ with r.e. complement. We denote the cosimple isols by $\Lambda_{z}$. By Theorem 56 of [4] $\Lambda_{z}$ is closed under isolic addition and predecessors (i.e., is an ideal in $\Lambda$ ) so that the notions of the previous sections are applicable to $\Lambda_{z}$. Here we show that if $\mathscr{F}$ is the sentence (2.6) and $E \vDash \mathscr{F}$ then $\Lambda_{Z} \vDash \mathscr{F}$.

Except for minor differences we use the terminology of $\S 3$. Let $\bar{X}=\left(X_{1}, \cdots, X_{p}\right)$ be a vector of cosimple isols satisfying the hypothesis $H$ of $\mathscr{F}$. Let $\omega$ be the even integers, and $\omega_{i}, 1 \leqq i \leqq p$ a partition of $\omega$ into infinite recursive subsets. Choose $\xi \subset \omega$ such that $\xi_{i}=$ $\xi \cap \omega_{i}$ represents $X_{i}$ and $\omega_{i}-\xi_{i}$ is r.e.. It is clear that this choice can be made since if $\xi_{i}^{\prime}$ is a representative of $X_{i}$ with r.e. complement and $f$ is a one-to-one recursive function mapping $E$ onto $\omega_{i}$ then $\xi_{i}=f\left(\xi_{i}^{\prime}\right)$ satisfies our requirements. Define complete $H$-chain as in $\S 3$ and let $S$ be the set of all finite complete $H$-chains. As mentioned in $\S 3, S$ is r.e. and hence can be enumerated by a recursive function $\gamma(m)$.

Let $\theta$ be the odd integers, and $\theta_{i}, 1 \leqq i \leqq r$ a partition of $\theta$ into infinite recusive subsets. We define a partial recursive function $h$ by the following induction. Suppose $h(\gamma(m))$ has been defined for $m<n$. Let $x_{i}=\left|\gamma(m) \cap \omega_{i}\right|$. Clearly the vector $\bar{x}=\left(x_{1}, \cdots, x_{p}\right)$ satisfies $H$ so there must be a vector of integers $\bar{y}=\left(y_{1}, \cdots, y_{r}\right)$ such that $(\bar{x}, \bar{y})$ satisfies the conclusion $C$ of $\mathscr{F}$. Let $\delta \subset \theta$ be the first finite set in the canonical enumeration of finite sets such that $\left|\delta \cap \theta_{i}\right|=$ $y_{i}$ and $\delta$ is disjoint from $h(\gamma(m))$ for $m<n$. Set $h(\gamma(n))=\delta$. Since $\delta$ can be effectively be found, $h$ is partial recursive. As in $\S 3$, finite functions $g_{i}$ can be effectively found such that $\gamma \cup h(\gamma)$ is a finite complete $C$-chain. Let $\eta=\bigcup\{h(\gamma) \mid \gamma \in S$ and $\gamma \subset \xi\}$ and $\eta_{i}=\eta \cap \theta_{i}$. 
Then as in $\S 3$, if $\eta_{i}$ is a representative of $Y_{i}$, and $\bar{Y}=\left(Y_{1}, \cdots, Y_{r}\right)$ then $\bar{Y}$ is a vector of isols such that $(\bar{X}, \bar{Y})$ satisfies $C$. It only remains to show that each $Y_{i}$ is cosimple. Let $\zeta=\bigcup\{h(\gamma) \mid \gamma \in S\}$. $\zeta$ is r.e. and since $\omega-\xi$ is r.e. so is $\zeta-\eta=\bigcup\{h(\gamma) \mid \gamma \in S$ and $\gamma \cap$ $(\omega-\xi) \neq \varnothing\}$. Let $q$ be a one-to-one recursive function mapping $E$ onto $\zeta_{i}=\zeta \cap \theta_{i}$. Then $E=q^{-1}\left(\eta_{i}\right) \cup q^{-1}\left(\zeta_{i}-\eta_{i}\right)$ and $q^{-1}\left(\eta_{i}\right)$ is a representative of $Y_{i}$ whose complement $q^{-1}\left(\zeta_{i}-\eta_{i}\right)$ is r.e.. Thus $Y_{i} \in \Lambda_{Z}$ which completes our proof.

There is also a converse to the $A E$ metatheorem for cosimple isols. Here we show that if $\mathscr{F}$ is the sentence (2.8) and $\Lambda_{Z} \vDash \mathscr{F}$ then some Horn reduct of $\mathscr{F}$ is true in $E$.

By the methods of $\S 4$ it will suffice to find a countable collection of pairwise incomparable indecomposable cosimple isols. Although it is possible to give a direct construction of such a collection, we prefer to obtain the result from existing literature. A set $\xi \subset E$ is maximal (cf. [14]) if $\xi$ is r.e. and for any r.e. set $\omega$, exactly one of $\omega \cap(E-\zeta)$ and $(E-\omega) \cap(E-\zeta)$ is infinite. Clearly if $\xi$ is maximal then its complement is a representative of an indecomposable cosimple isol. By [5] maximal sets exist. By theorem 5.1 of [7] the Turing degree of a maximal set contains a countably infinite collection of maximal sets having pairwise incomparable many-one degrees. By theorem 58 of [4] if $\alpha$ and $\beta$ are infinite sets with r.e. complements, and the RET of $\alpha$ is $\leqq$ the RET of $\beta$ then $\alpha$ is one-to-one reducible to $\beta$. Combining those facts gives the desired result.

\section{REFERENCES}

1. R. Bradford, Cardinal addition and the axiom of chioce, Ph. D. Thesis, University of California, Berkeley, California, 1965, also Ann. Math. Logic, 3 (1971), 111-196.

2. J. C. E. Dekker, A non-constructive extension of the number system, Parts $I$ and II, J. Symbolic Logic, 20 (1955), 204-205.

3. Congruences in isols with a finite modulus, Mathematische Zeitschrift, 70 (1958), 357-373.

4. J. C. E. Dekker and J. Myhill, Recursive equivalence types, University of California Publications in Mathematics (N.S.) 3, No. 3 (1960), 67-214.

5. R. M. Friberg, Three theorems on recursive enumeration, J. Symbolic Logic, 23 (1958), 309-316.

6. - The uniqueness of finite division for recursive equivalence types, Mathematische Zeitschrift, 75 (1961), 3-7.

7. M. Lerman, Recursive functions modulo comaximal sets, Ph. D. Thesis, Cornell University, 1968.

8. A. B. Manaster, $A$ theorem on the decomposition of linear forms for the isols, Notices of the Amer. Math. Soc. II (1964), 527.

9. — Higher-order indecomposable isols, Trans. Amer. Math. Soc., 125 (1966), 363-383.

10. The failure of Tarski's decomposition of linear forms theorem in the $R E T s$, in preparation.

11. A. Nerode, Additive relations among recursive equivalence types, Mathematische 
Annalen, 159 (1965), 329-343.

12. A. Nerode and A. B. Manaster, A universal embedding property of the RETs, J. Symbolic Logic, 35 (1970), 51-59.

13. M. Presburger, Über die Vollständigkeit eines gewissen Systems der Arithmetik ganzer Zahlen, in welchem die Addition als einzige Operation hervortritt, Sprawozdania z I Kongresu Matematykow Krajów Słowianskich, Warszawa (1929), 92-101 and 395.

14. H. Rogers Jr., Theory of Recursive Functions and Effective Computability, McGrawHill, New York, 1967.

15. A. Tarski, Cancellation laws in the arithemetic of cardinals, Fundamenta Mathematicae, 36 (1949), 77-92.

16. — Cardinal algebras, Oxford University Press, New York, 1949.

Received June 21, 1971 and in revised form April 11, 1972. Much of this work is part of a thesis submitted to Cornell University by the second author in 1965 . He wishes to acknowledge many stimulating hours of discussion with and the continuing encouragement of his advisor, Professor Anil Nerode. He also wishes to acknowledge the financial support for this research of the National Science Foundation.

Rutgers-The State UNIVERsity

AND

University of California, SAN Diego 


\section{PACIFIC JOURNAL OF MATHEMATICS}

\section{EDITORS}

\author{
H. SAMELSON \\ Stanford University \\ Stanford, California 94305 \\ C. R. HOBBY \\ University of Washington \\ Seattle, Washington 98105
}

\section{J. DugundjI}

Department of Mathematics University of Southern California Los Angeles, California 90007

\section{RICHARD ARENS}

University of California

Los Angeles, California 90024

\section{ASSOCIATE EDITORS}
E. F. BECKENBACH
B. H. NeumanN
F. WOLF
K. YosHIDA

\section{SUPPORTING INSTITUTIONS}

\author{
UNIVERSITY OF BRITISH COLUMBIA \\ CALIFORNIA INSTITUTE OF TECHNOLOGY \\ UNIVERSITY OF CALIFORNIA \\ MONTANA STATE UNIVERSITY \\ UNIVERSITY OF NEVADA \\ NEW MEXICO STATE UNIVERSITY \\ OREGON STATE UNIVERSITY \\ UNIVERSITY OF OREGON \\ OSAKA UNIVERSITY
}

\author{
UNIVERSITY OF SOUTHERN CALIFORNIA \\ STANFORD UNIVERSITY \\ UNIVERSITY OF TOKYO \\ UNIVERSITY OF UTAH \\ WASHINGTON STATE UNIVERSITY \\ UNIVERSITY OF WASHINGTON \\ ${ }^{*} \stackrel{*}{*}{ }^{*}$
AMERICAN MATHEMATICAL SOCIETY
NAVAL WEAPONS CENTER
}

The Supporting Institutions listed above contribute to the cost of publication of this Journal, but they are not owners or publishers and have no responsibility for its content or policies.

Mathematical papers intended for publication in the Pacific Journal of Mathematics should be in typed form or offset-reproduced, (not dittoed), double spaced with large margins. Underline Greek letters in red, German in green, and script in blue. The first paragraph or two must be capable of being used separately as a synopsis of the entire paper. The editorial "we" must not be used in the synopsis, and items of the bibliography should not be cited there unless absolutely necessary, in which case they must be identified by author and Journal, rather than by item number. Manuscripts, in duplicate if possible, may be sent to any one of the four editors. Please classify according to the scheme of Math. Rev. Index to Vol. 39. All other communications to the editors should be addressed to the managing editor, Richard Arens, University of California, Los Angeles, California, 90024.

50 reprints are provided free for each article; additional copies may be obtained at cost in multiples of 50 .

The Pacific Journal of Mathematics is published monthly. Effective with Volume 16 the price per volume (3 numbers) is $\$ 8.00$; single issues, $\$ 3.00$. Special price for current issues to individual faculty members of supporting institutions and to individual members of the American Mathematical Society: $\$ 4.00$ per volume; single issues $\$ 1.50$. Back numbers are available.

Subscriptions, orders for back numbers, and changes of address should be sent to Pacific Journal of Mathematics, 103 Highland Boulevard, Berke'ey, California, 94708.

PUBLISHED BY PACIFIC JOURNAL OF MATHEMATICS, A NON-PROFIT CORPORATION

Printed at Kokusai Bunken Insatsusha (International Academic Printing Co., Ltd.), 270, 3-chome Totsuka-cho, Shinjuku-ku, Tokyo 160, Japan. 


\section{Pacific Journal of Mathematics}

\section{Vol. 43, No. $3 \quad$ May, 1972}

Max K. Agoston, An obstruction to finding a fixed point free map on a manifold.... 543

Nadim A. Assad and William A. Kirk, Fixed point theorems for set-valued mappings

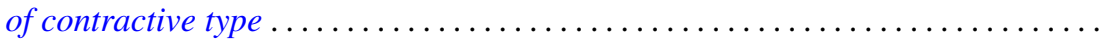

John Winston Bunce, Characterizations of amenable and strongly amenable

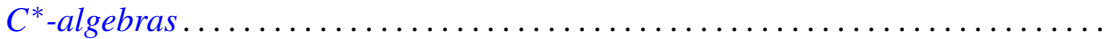

Erik Maurice Ellentuck and Alfred Berry Manaster, The decidability of a class of

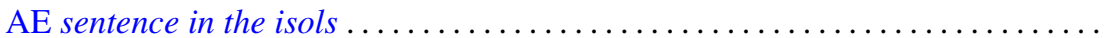

U. Haussmann, The inversion theorem and Plancherel's theorem in a Banach

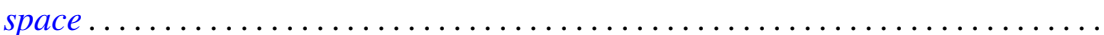

Peter Lawrence Falb and U. Haussmann, Bochner's theorem in infinite dimensions.

Peter Fletcher and William Lindgren, Quasi-uniformities with a transitive base ..... Dennis Garbanati and Robert Charles Thompson, Classes of unimodular abelian

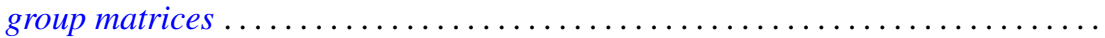

Kenneth Hardy and R. Grant Woods, On c-realcompact spaces and locally bounded normal functions

Manfred Knebusch, Alex I. Rosenberg and Roger P. Ware, Grothendieck and Witt rings of hermitian forms over Dedekind rings .......................

George M. Lewis, Cut loci of points at infinity.

Jerome Irving Malitz and William Nelson Reinhardt, A complete countable $L_{\omega_{1}}^{Q}$ theory with maximal models of many cardinalities . . . . . . . . . . . . . . . . .

Wilfred Dennis Pepe and William P. Ziemer, Slices, multiplicity, and Lebesgue

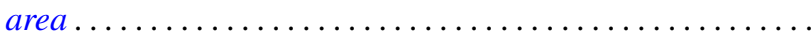

Keith Pierce, Amalgamating abelian ordered groups . .

Stephen James Pride, Residual properties of free groups . . . . . . . . . . . . . 725

Roy Martin Rakestraw, The convex cone of n-monotone functions .

T. Schwartzbauer, Entropy and approximation of measure preserving transformations .

Peter F. Stebe, Invariant functions of an iterative process for maximization of a polynomial...

Kondagunta Sundaresan and Wojbor Woyczynski, L-orthogonally scattered

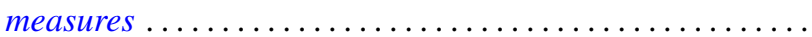

Kyle David Wallace, $C_{\lambda}$-groups and $\lambda$-basic subgroups $\ldots \ldots \ldots$

Barnet Mordecai Weinstock, Approximation by holomorphic functions on certain

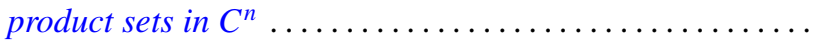

Donald Steven Passman, Corrections to: "Isomorphic groups and group rings”.

Don David Porter, Correction to: "Symplectic bordism, Stiefel-Whitney numbers, and a Novikov resolution"

John Ben Butler, Jr., Correction to: “Almost smooth perturbations of self-adjoint operators".

Constantine G. Lascarides, Correction to: "A study of certain sequence spaces of Maddox and a generalization of a theorem of Iyer" ...... ...

George A. Elliott, Correction to: "An extension of some results of takesaki in the reduction theory of von neumann algebras" ......................... 